\title{
Reliability Study of DEPP Functionally Graded Actuators
}

\author{
Paul W. Alexander ${ }^{*}$, Diann Brei ${ }^{\dagger}$, and John W. Halloran ${ }^{\ddagger}$ \\ University of Michigan, Ann Arbor, Michigan, 48109
}

\begin{abstract}
The infiltration of piezoceramic actuation technology into mainstream industrial applications has been limited. While there are many factors that can be cited, one of the main obstacles to the adoption of this technology has been reliability. For example, internally leveraged architectures rely on strain gradients across the device, realized by bonding together homogeneous material layers, to amplify the deflection of the bulk piezoceramic. Unfortunately, this commonly introduces large stress gradients and discontinuities along with peeling/shearing stresses at these material interfaces, resulting in delamination within $10^{5}$ to $10^{6}$ cycles, well below the range required by most industries. Functionally Graded Piezoceramic (FGP) technology alleviates these issues by eliminating the need for bonds altogether by spatially grading specific material properties within a monolithic piezoceramic, producing similar, but slightly lowered, strain gradients to those found in conventional internally leveraged actuators. The Dual Electro/Piezo Property (DEPP) gradient technique produces FGP with a synergistic gradient of the electric permittivity and piezoelectric properties, simultaneously increasing the efficiency and deformation capabilities compared to other gradient schemes. This paper presents an initial study of the reliability and the deflection amplification costs associated with DEPP FGP actuators. Numeric and analytic models were derived to investigate the inherent tradeoff between internal stress reduction and displacement costs that arise from graded fabrication. To validate the premise that lifetime is increased by DEPP FGP fabrication, two types of fatigue experiments were conducted by cyclic activation of prototypes below resonance and at resonance. These initial tests revealed promising results, providing evidence of lifetimes well above $10^{10}$ cycles for DEPP FGP actuators, potentially overcoming the reliability limitations that have historically plagued conventional piezoceramic actuators and hindered their acceptance in commercial applications.
\end{abstract}

\section{Nomenclature}

$a \quad=$ neutral axis position

$C_{i} \quad=$ material property gradient integral

$c \quad=$ material stiffness

$d \quad=$ piezoelectric strain coefficient

$D \quad=$ electric displacement

$E \quad=$ electric field

$H \quad=$ electric enthalpy density

$l_{i} \quad=$ beam dimension in the $x_{i}$ direction

$l=$ specimen length

$S \quad=$ strain

$T=$ stress

$t=$ specimen thickness

$u_{i} \quad=$ displacement component

$U \quad=$ internal energy

\footnotetext{
* Researcher, General Motors R \& D, Mail Code: 480-106-256, 30500 Mound Road, Warren, MI 48090-9055, AIAA Member.

${ }^{\dagger}$ Associate Professor, Department of Mechanical Engineering, 2350 Hayward Street, Ann Arbor, MI 48109-2125, AIAA Member.

${ }^{*}$ Professor, Department of Materials Science and Engineering, 2300 Hayward Street, Ann Arbor, MI 48109-2136.
} 


$\begin{array}{ll}V_{i} & =\text { electric potential } \\ w & =\text { specimen width } \\ x_{i} & =\text { spatial coordinate } \\ Y & =\text { Young's modulus } \\ \Delta V & =\text { electric potential difference } \\ \varepsilon & =\text { electric permittivity }\end{array}$

\section{Introduction}

TNTERNALLY leveraged piezoceramic actuation architectures rely on material variances across the structure to amplify the strain. Most of these conventional actuators are physically realized by bonding together homogeneous material layers that have dissimilar material properties, creating stress and strain gradients in the actuator that results in amplified deflection. ${ }^{1-4}$ Unfortunately, this commonly introduces peeling and shearing stresses at the same location where the stress gradients reach their maximum. For these actuators, delamination typically occurs within $10^{5}$ to $10^{6}$ cycles, well below the range required by most industries. ${ }^{5,6}$ The failure modes of these actuation architectures are difficult to identify, research, and address since it is dependent upon the individual design. Most of the modeling effort has focused on capturing the stress distribution within conventional actuators. ${ }^{7-14}$ Their bonded construction results in extreme stress states at the ends of the structure, facilitating crack initiation at the interface between adjacent material layers. There has been some limited studies into the reliability of piezoceramic actuators in general, concentrating on common actuation architectures like stacks and layered benders. ${ }^{12-17}$

An elegant approach to solve these reliability problems is to grade the properties of a monolithic piezoceramic, producing Functionally Graded Piezoceramics (FGP). ${ }^{18-27}$ These monolithic materials exploit material gradients to produce similar deformations to conventional actuation architectures, yet preclude many of the common architecture failure mechanisms. By removing the adhesive bonding layers, delamination inducing stresses are eliminated. In addition, the internal stress profile is reduced and smoothed so that the ceramic material is less likely to experience stress concentrations that could lead to crack initiation and propagation. However, these promised reliability enhancements come at the sacrifice of actuation authority, ${ }^{28}$ with the same continuous material grading responsible for the improved stress state also affecting the strain gradient within the material. The Dual Electro/Piezo Property (DEPP) grading technique addresses this by synergistically varying both the piezoelectric coefficients and the electrical permittivity of the material, focusing the bulk of the electric field in the most active material, efficiently amplifying the induced bending deformations at lower driving voltages. ${ }^{29}$

This paper presents an initial study of the reliability and the amplification cost associated with DEPP FGP actuators. Numerical models and analytical models were derived to investigate the tradeoff between the reduction in stress and displacement. To validate the premise that lifetime is increased by DEPP FGP fabrication, two types of fatigue experiments were conducted by cyclic activation of fatigue specimens below resonance and at resonance. Below resonance testing was preformed using a bimodally graded DEPP FGP fabricated using traditional powder pressing methods. The lower frequency sine wave used in this fatigue experiment more closely approximates quasi-static operational conditions similar to what actuators will typically experience in commercial applications. More demanding tests at resonance were conducted using a pair of geometrically similar specimens tuned to exhibit identical resonant frequencies: a monolithic, bimodally graded DEPP FGP and a conventional bender created by bonding together the same two gradient materials. While this reliability study was not extensive, it does indicate a significant improvement in reliability for DEPP FGP (up to the level required by industry) compared to conventional technologies.

\section{FGP Performance/Reliability Tradeoffs}

To assess the impact in the reduction of internal stresses on the both the reliability and deflection capabilities of continuously graded and discretely layered actuators, a simulation study utilizing numeric and analytic models was conducted. Analytic models were chosen to exactly represent continuous gradients, in this case linear, and numeric models were chosen to exactly represent discretely layered gradients and assess the impact of linear assumptions in the analytic model. All of the models were based on a cantilevered, $30 \mathrm{~mm}$ long by $4 \mathrm{~mm}$ wide by $2 \mathrm{~mm}$ thick beam activated by a $400 \mathrm{~V}$ electric potential. Each of the models predicts both the longitudinal stress profile and displacement performance of the actuator under free deflection conditions. 


\section{A. Analytic Modeling}

The detailed derivation of the FGP beam's equations of motion is given by Alexander, Brei, and Halloran ${ }^{30}$ and summarized here for clarity. The deformations of an unloaded piezoceramic beam functionally graded through the thickness (Fig. 1) were determined using its internal energy density $(U)$,

$$
U=H+D E,
$$

where $H$ is the electric enthalpy density, $D$ is the electric displacement, and $E$ is the applied electric field. Using the definition of the electric enthalpy density,

$$
H=\frac{1}{2} c_{i j k l}^{E} S_{i j} S_{k l}-S_{i j} e_{k i j} E_{k}-\frac{1}{2} \varepsilon_{i j}^{S} E_{i} E_{j},
$$

and Hamilton's principle of minimal energy, the variation of the total internal energy ( $U^{\text {Total }}$ ) must be equal to zero,

$$
\delta U^{\text {Total }}=\int_{v}\left\{\frac{1}{2} c_{i j k l}^{E} \delta S_{i j} S_{k l}+\frac{1}{2} c_{i j k l}^{E} S_{i j} \delta S_{k l}-c_{n m i j}^{E} \delta S_{i j} d_{n m j} E_{k}\right\} d v=0,
$$

with $S$ denoting the material strain, $c$ the material stiffness (which can be represented by the Young's modulus, $Y$ ), $d$ the piezoelectric strain coefficient, and the superscript $E$ indicating the constant electric field conditions prescribed when using the FGP as an actuator. Assuming Bernoulli-Euler bending conditions, the deflection components $\left(u_{i}\right)$ for an FGP beam when electrically activated are

$$
\begin{gathered}
u_{1}=\left(\frac{C_{2} C_{5}-C_{3} C_{4}}{C_{2}{ }^{2}-C_{1} C_{3}}\right) x_{1}-\left(\frac{C_{1} C_{5}-C_{2} C_{4}}{C_{2}{ }^{2}-C_{1} C_{3}}\right) x_{1}\left[x_{3}-a\right], \\
u_{2}=0, \\
u_{3}=\frac{1}{2}\left(\frac{C_{1} C_{5}-C_{2} C_{4}}{C_{2}{ }^{2}-C_{1} C_{3}}\right) x_{1}{ }^{2} .
\end{gathered}
$$

where $x_{1}$ and $x_{3}$ denote the length and thickness coordinates of the beam respectively and $a$ is the distance to the neutral axis, calculated using the relation

$$
\int_{0}^{a}\left\{Y_{11}\left(x_{3}\right)\right\} d x_{3}=\int_{a}^{l_{3}}\left\{Y_{11}\left(x_{3}\right)\right\} d x_{3},
$$

with $l_{3}$ denoting the beam thickness and $Y$ the material Young's modulus. The $C_{i}$ terms in Equations (4)-(6) are thickness integrals that capture the material gradient within the actuator,

$$
\begin{gathered}
C_{i}=\int_{0}^{l_{3}}\left\{\left[x_{3}-a\right]^{i-1} Y_{11}\left(x_{3}\right)\right\} d x_{3} \text { for } i=1 \text { to } 3, \\
C_{i}=\int_{0}^{l_{3}}\left\{\left[x_{3}-a\right]^{i-4} d_{31}\left(x_{3}\right) E_{3}\left(x_{3}\right) Y_{11}\left(x_{3}\right)\right\} d x_{3} \text { for } i=4 \text { and } 5,
\end{gathered}
$$

including the variation in the modulus and piezoelectric strain coefficient $(d)$, and the complex electric field profile

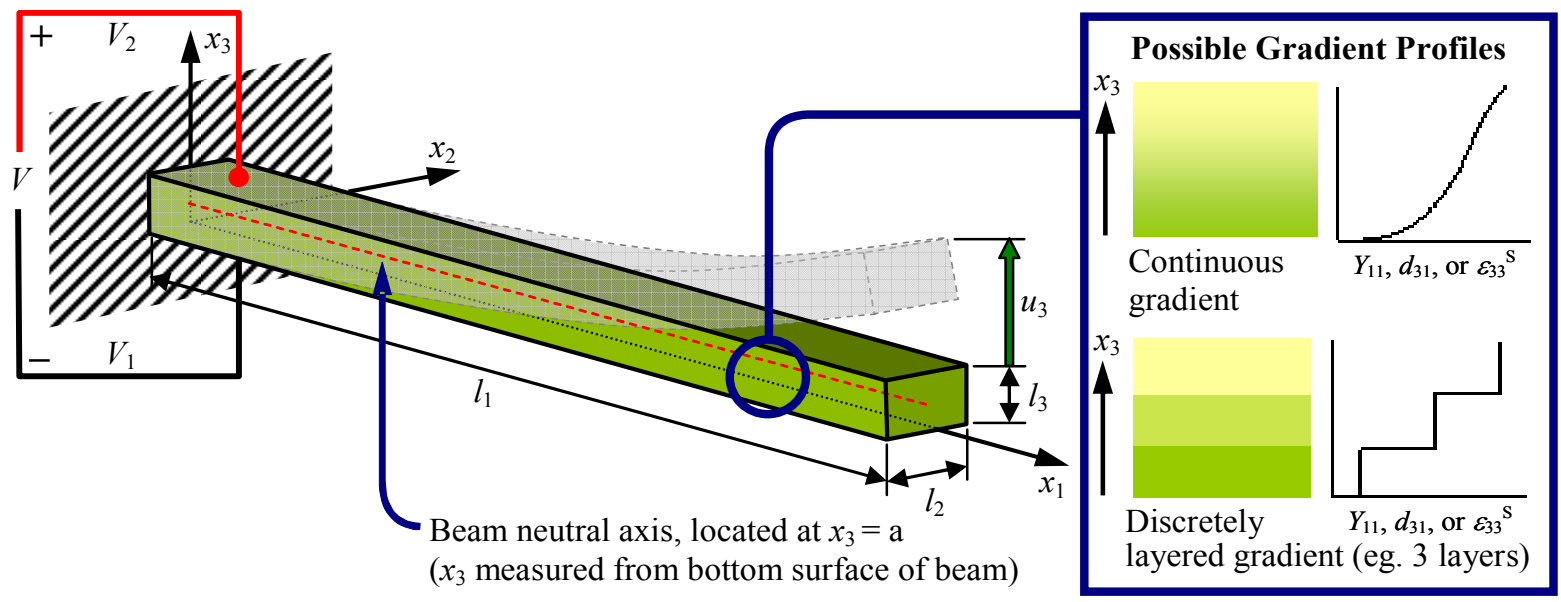

Figure 1. FGP Cantilever Beam with Thickness Graded Material Properties. 
that a graded material will experience $(E)$. This activating field depends on the permittivity $(\varepsilon)$ gradient within the FGP and the applied electric potential $\left(\Delta V=V_{2}-V_{1}\right)$,

$$
E_{3}\left(x_{3}\right)=\frac{-\Delta V}{\varepsilon_{33}\left(x_{3}\right) \int_{0}^{l_{3}}\left\{\frac{1}{\varepsilon_{33}\left(x_{3}\right)}\right\} d x_{3}} .
$$

The profile must be accurately captured during modeling in order to correctly predict the stress distribution in the actuator as well as its deflection capabilities. ${ }^{30}$

For reliability assessment, the internal stresses during activation are critical. The stress $(T)$ profile within these FGP beams can be predicted as a function of strain $(S)$ and electric field $(E)$ using the constitutive equation,

$$
T=c^{E} S-c^{E} d E
$$

The strain within the beam is defined using the displacement components $\left(u_{i}\right)$ and the standard strain definition,

$$
S_{i j}=\frac{1}{2}\left[\frac{\partial u_{i}}{\partial x_{j}}+\frac{\partial u_{j}}{\partial x_{i}}\right]
$$

Substitution of Equations (4)-(6) into the strain equation results in a single strain component, which gives rise to the longitudinal stress along the beam length $\left(T_{11}\right)$,

$$
T_{11}\left(x_{3}\right)=Y_{11}\left(x_{3}\right)\left\{\left(\frac{C_{2} C_{5}-C_{3} C_{4}}{C_{2}^{2}-C_{1} C_{3}}\right)-\left(\frac{C_{1} C_{5}-C_{2} C_{4}}{C_{2}^{2}-C_{1} C_{3}}\right)\left[x_{3}-a\right]\right\}-Y_{11}\left(x_{3}\right) d_{31}\left(x_{3}\right) E_{3}\left(x_{3}\right) .
$$

This stress is highly dependent on the material gradient within the FGP, varying with the material properties and electric field across the beam thickness. The theoretic linear material gradient was substituted into this model to predict both the deformed actuator shape, $u_{3}\left(x_{1}\right)$ - (Eq. (6)), and the stress profile, $T_{11}\left(x_{3}\right)$ - (Eq. (13)).

\section{B. Numeric Modeling}

While the analytic model is an efficient tool for predicting the stress within a continuously graded FGP, it was based upon a linear constitutive model neglecting the piezoelectric effects in the width and thickness directions. Thus, to assess the cost in simplification, numeric models were constructed that fully capture the three dimensional state along with one dimensional models with their piezoelectric properties in the two (width) and three (thickness) direction suspended, similar to the assumptions made when deriving the continuous analytic model. Since numeric models are constructed using elements, they are useful in accurately modeling the stepped property gradients of layered actuators which would have to be piecewise integrated into the analytical model. In contrast, the analytical model captures better continuous gradients that must be approximated with many elements within the numeric model in order to achieve the same degree of accuracy.

The discretely layered actuators to be used for the tradeoff investigation were constructed using ABAQUS CAE v6.3, consisting of 20-node quadratic piezoelectric brick elements using linear piezoelectric theory (C3D20E) in a cantilever configuration (Fig. 2). Both the one dimensionally and three dimensionally modeled actuators included stepped gradients composed of two, four, eight, and twenty material layers covering the same range of variation in the material stiffness, density, dielectric constant, and piezoelectric coefficients. The free deflection of the actuators was obtained by applying an electric potential to the top and bottom surfaces of the models and calculating the deflection in the $x_{3}$ direction along the beam's center. The predicted longitudinal stress was measured across the beam's thickness a few elements from the mounted end at the center of the beam's width under the same activation conditions.

\section{Model Comparison}

Figure 3 compares the three modeling approaches. As expected, for discretely layered beams there is a $25 \%$ difference in the one dimensional and three dimensional numeric stress models arising from the additional piezoelectric strains and Poisons effects. As the number

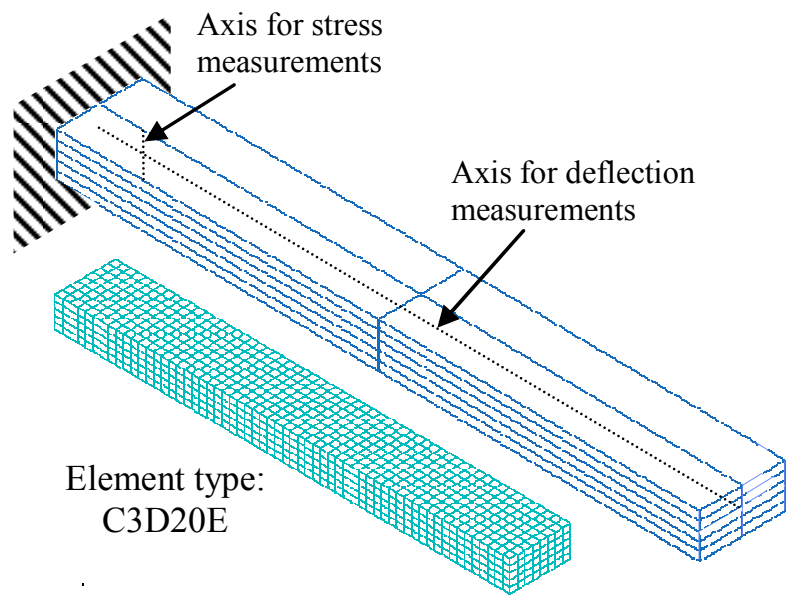

Figure 2. Five-Layer Finite Element Model of a Thickness Graded FGP Beam. 
of discrete layers increases and the stress profile smoothes, the discrepancy becomes less pronounced with only $0.13 \mathrm{MPa}$ difference at 20 layers and less if extrapolated to the a continuous gradient. However, the deflection predictions are never different by more than $0.5 \%$. Thus, the one dimensional assumptions of the analytic continuous models are reasonable, especially for actuators incorporating a large number of material layers or continuous material gradients.

More importantly, this data illustrates how dramatic the stress can be reduced when only a few extra layers are introduced into the actuator, with the maximum stress dropping by $50 \%$ for a penalty of only $2.3 \mu \mathrm{m}$ of deflection (15\%) with two layers compared to four layers. In applications where device longevity is key, such a tradeoff can be critical, determining whether piezoceramic actuation could be within a solution space or relegated as an infeasible technology. Coupling these findings with the ability to specifically tailor material gradients anywhere between the extremes of linear or bimodal profiles, it is realistic to conceive of custom FGP actuators with specifically designed material gradients that balance these tradeoffs in a manner that best suits the demands of the application.

\section{Displacement Costs}

Figure 4 gives the predicted displacements in the $x_{3}$ direction along the beams' length coordinate for the analytic model of the continuous linearly graded FGP and the numeric models of the layered, discretely stepped FGP. As expected, the inclusion of additional material layers in the numeric models with transitional material compositions reduced the deflection capabilities of the FGP beams. Even though the displacement drops from $15.3 \mu \mathrm{m}$ for the original two step model to $10.9 \mu \mathrm{m}$ for the twenty step model, a reduction of $28.7 \%$, the degradation in actuator performance with additional gradient steps is much less dramatic when comparing models with a larger number of gradient steps. When model complexity has reached seven or eight layers, the model prediction varies by only a slight amount, $0.2 \mu \mathrm{m}$, with any additional gradient layers yielding similarly small reductions in predicted displacement. The analytic linear model predicts $10.3 \mu \mathrm{m}$ of deflection, a reduction of $32.5 \%$ compared to the two composition beam. This reduction in deflection is the upper bound on the performance cost of grading.

\section{E. Stress Reductions}

Figure 5 evaluates the predicted longitudinal stress $\left(T_{11}\right)$ across the beam thickness for the different FGP models. It displays the dramatic spikes and discontinuities for the layered models where dissimilar materials are adjacent to each other. Large material gradient steps result in higher tensile stress levels, reaching a maximum of $1.87 \mathrm{MPa}$ for the two layered case, which is lowered to a peak stress of only $0.45 \mathrm{MPa}$ for the twenty layer model, a reduction of $76.1 \%$. The inclusion of additional gradient layers with intermediate material properties greatly reduces the peak

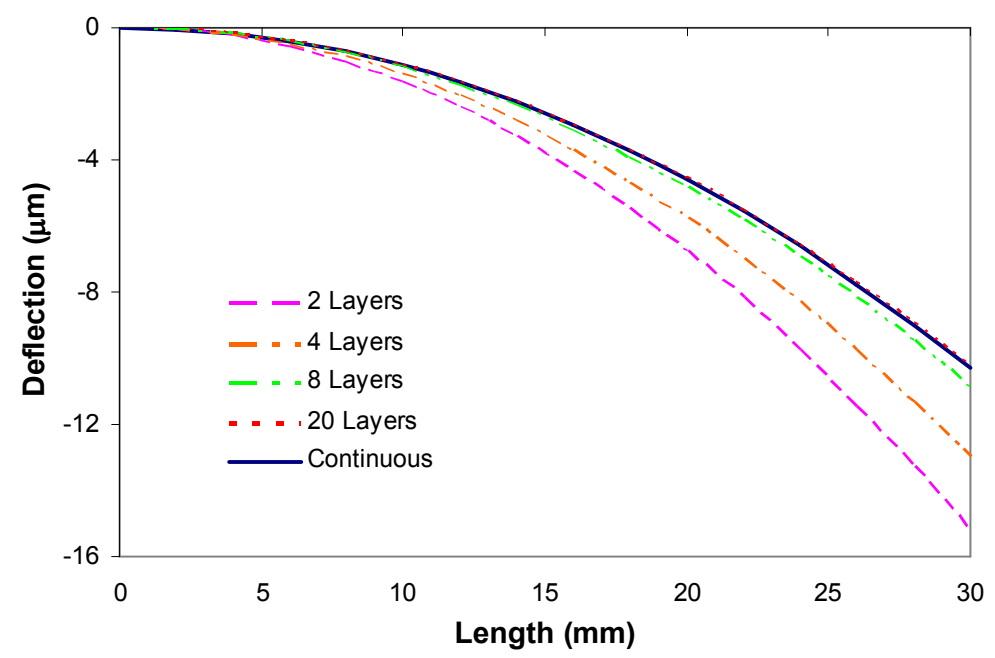

Figure 4. Predicted Displacement along the Length of Layered and Continuously Graded Beams. 
stress values and produces a more uniform stress distribution within the actuator. Adding more and more material layers to the numeric models causes the predictions to approach the limiting case, represented by the linearly graded analytic model. A continuous liner gradient yields an $86.2 \%$ reduction in the maximum internal longitudinal stress experienced during activation when compared to the conventional two layered actuator. Not only is the magnitude of the internal longitudinal stresses reduced, but the discontinuities within the stress profile, indicative of large peeling and shearing stresses, are replaced by a gently curving stress distribution with peak stresses at the top and bottom surfaces of the actuator. This dramatic longitudinal stress reduction

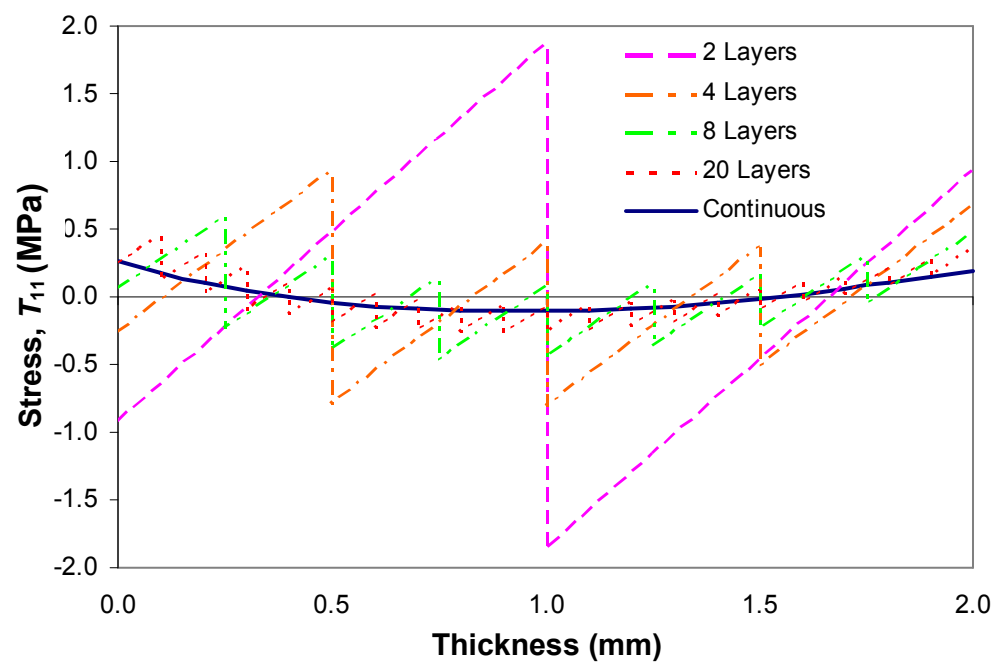

Figure 5. Predicted Longitudinal Stress Levels within Layered and Continuously Graded Beams. and the resulting elimination of shear and peeling stresses would undoubtedly extend the lifetime of actuation architectures that currently use a conventional, multiple layered construction approach. Thus, the $32.5 \%$ limitation in deflection capabilities is much more acceptable if it comes hand-in-hand with an $86.2 \%$ reduction in internal stress levels.

\section{Fatigue Testing DEPP FGP Actuators}

To validate the hypothesis that a reduced stress state within DEPP FGP leads to longer lifetimes, two different fatigue experiments were conducted. The fatigue specimens for these studies use the optimal DEPP material gradient, varying the composition from pure lead zirconate titanate (100 vol\% PZT) to a material doped with $20 \%$ barium titanate dielectric $(80 / 20 \mathrm{vol} \% \mathrm{PZT} / \mathrm{BT}){ }^{29}$ As an initial trial, a bimodally graded, powder pressed actuator was cycled below its resonance by sinusoidal excitation. The results of this experiment give a sense of the lifetime of the DEPP graded actuator in many commercial applications where the device will not be pushed to their resonant frequency. To directly compare the long-term performance of conventionally constructed actuators to graded actuators, a second test was conducted using a two layer conventionally constructed actuator and an analogous co-extruded, continuous, bimodally graded actuator. Both specimens were simultaneously tested, cyclically activated at their resonant frequency. These results show the fatigue performance of FGP at the more demanding resonant conditions and allow for direct comparison of the two actuation technologies to realistically gauge the benefits of graded construction.

\section{A. Below Resonance Testing}

For the first fatigue test, a bimodally graded, powder pressed prototype actuator was cyclically activated below the actuator resonance to quantify the potential lifetime improvement graded construction may afford under typical actuation conditions.

\section{Prototype}

Traditional powder pressing was selected for the first sample since it is a fast, simple, and reliable means for manufacturing basic functionally graded piezoceramics. ${ }^{19,20,26,27}$ The actuator for this test was powder pressed from the
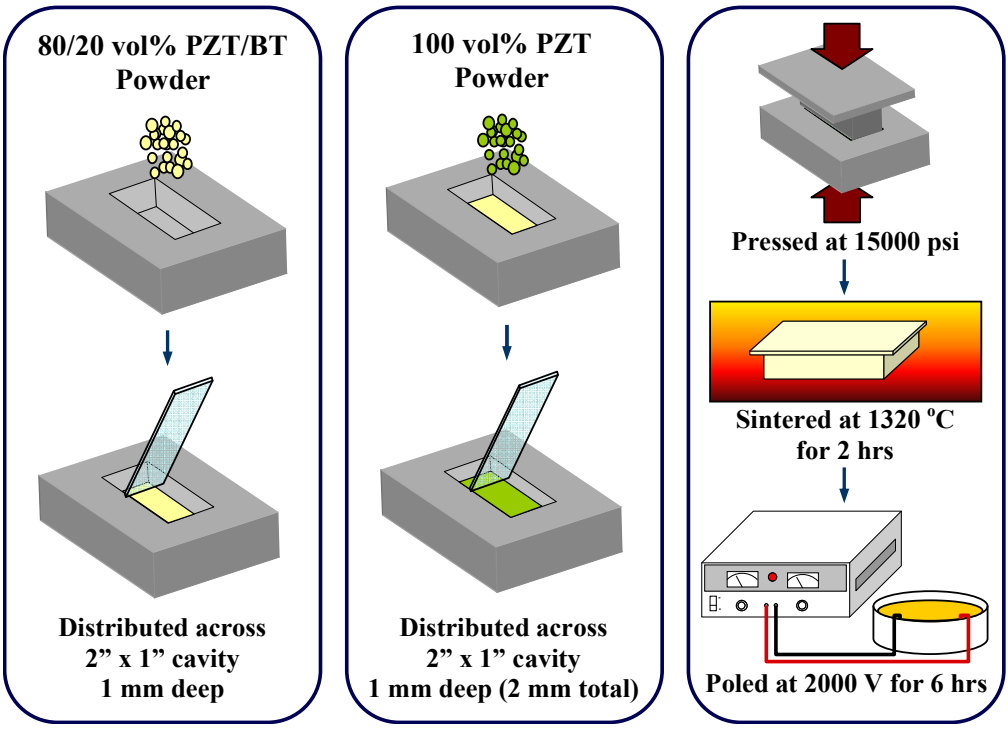

Figure 6. Overview of the Powder Pressing Process for DEPP FGP. 
two optimal DEPP powder compositions, $100 \mathrm{vol} \%$ PZT and 80/20 vol\% PZT/BT, producing a continuous gradient that is similar to a two composition discretely layered actuator in performance and internal stress levels. ${ }^{31}$ The DEPP powder pressing process (Fig. 6) combines equal amounts of the two powder compositions, layering one on top of the other in a 2" 1 " steel die compressed with $15,000 \mathrm{psi}$, forming a green ceramic plate approximately $2 \mathrm{~mm}$ thick. After sintering the sample at $180{ }^{\circ} \mathrm{C} / \mathrm{hr}$ to a maximum temperature of

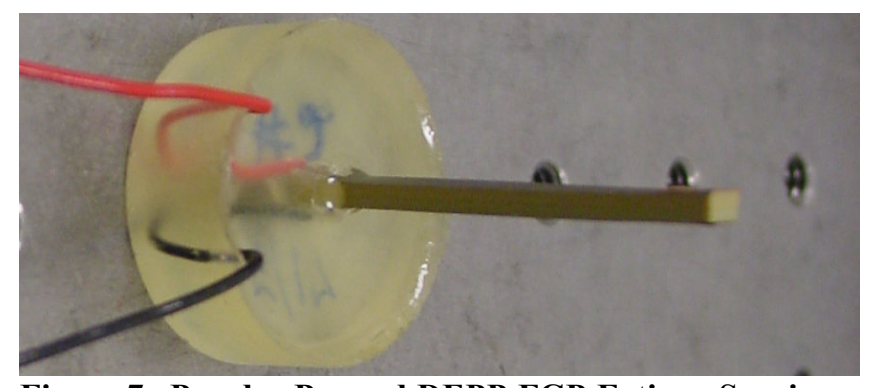

Figure 7. Powder Pressed DEPP FGP Fatigue Specimen $(l=32.66 \mathrm{~mm}, w=2.29 \mathrm{~mm}, t=2.31 \mathrm{~mm})$.
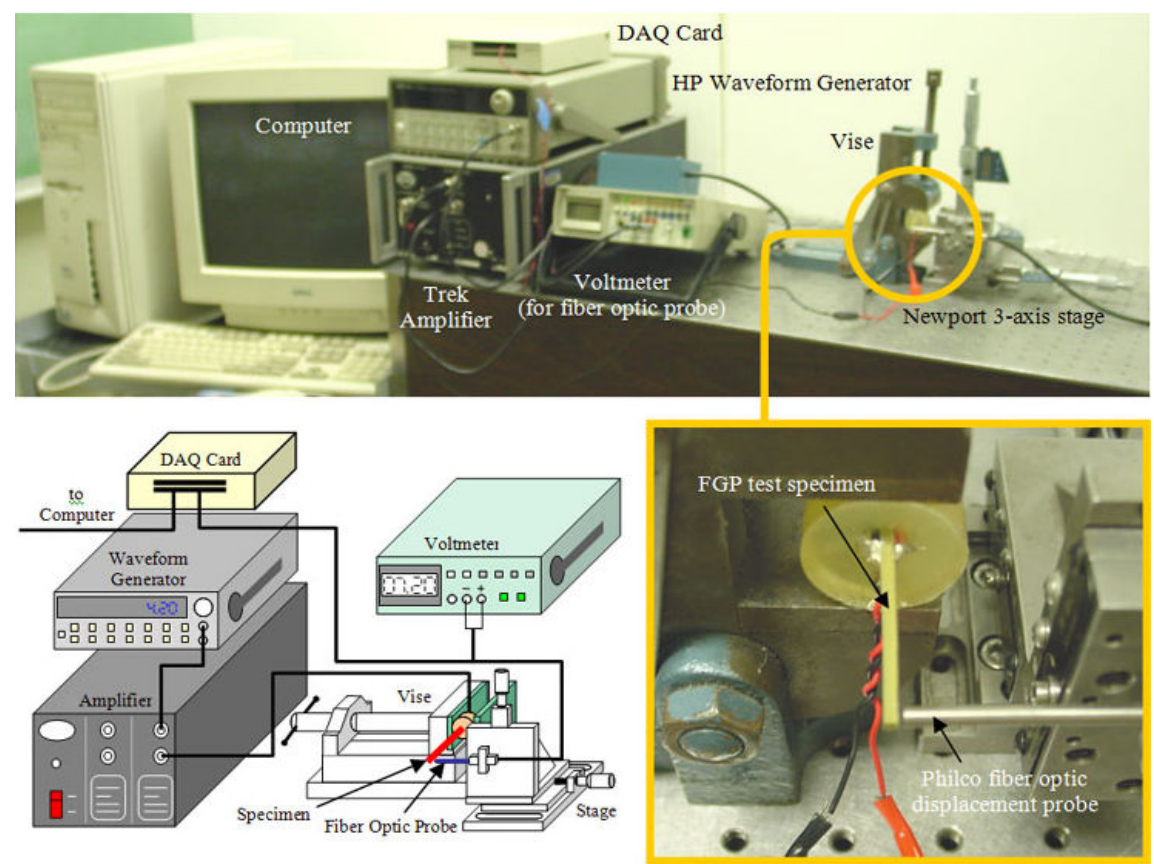

Figure 8. Test Set-Up for Initial DEPP FGP Fatigue Characterization.
$1320{ }^{\circ} \mathrm{C}$ that was maintained for two hours before the sample was cooled, the plate was cut into beams with a diamond saw and sputter coated with a $\mathrm{Au}-\mathrm{Pd}$ electrode then poled at $2000 \mathrm{~V}^{29}$ The resulting test specimens were approximately $32 \mathrm{~mm}$ long, $2 \mathrm{~mm}$ wide, and $2 \mathrm{~mm}$ thick (Fig. 7).

2. Experimental Procedure

The actuator was fatigued by subjecting it to a sinusoidal excitation of $450 \mathrm{~V}$ at $500 \mathrm{~Hz}$ supplied by a HP 33120A arbitrary waveform generator and Trek 50/750 amplifier (Fig. 8). The deflection of the actuator was measured every two hundred seconds by the fiber optic probe, acquired and recorded using a computer with National Instrument's LabView software. The applied driving potential was limited to about $65 \%$ of the maximum suggested driving electric field to ensure that changes in the performance of the actuator would be less likely due to degradation of the piezoelectric effect than structural failure of the device. Additionally, the activation frequency for this test was limited to approximately one half the resonant frequency of the FGP beam.

3. Results

The results from this study are given in Figure 9. The specimen did not fail for the duration of the fatigue test, but did have to be remounted because the original electrical leads detached from the actuator after $10^{7}$ cycles. The variation in the displacement readings early in the test are due to the electrical connection problems and the

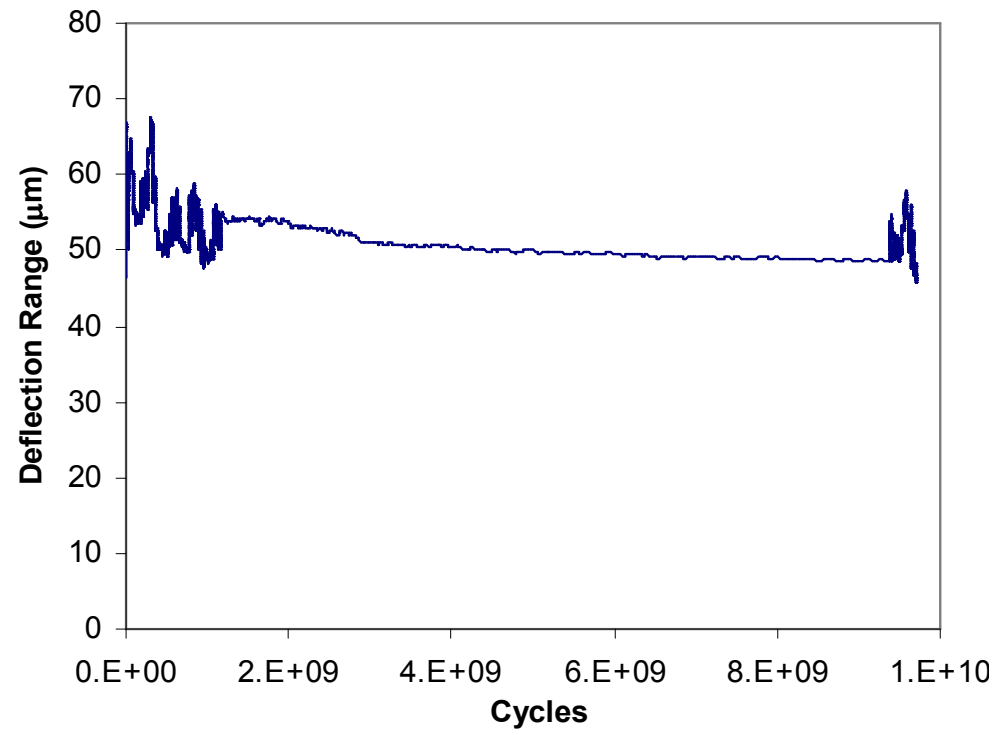

Figure 9. Long Term Deflection Performance of the Powder Pressed Bimodal DEPP FGP Actuator. 
actuator reaching a steady state temperature since testing was initial stopped at the end of the day and restarted on the following morning. Once new leads were attached the specimen using Tra-Con's BA-2902 silver based conductive epoxy and the remainder of the experiment was run uninterrupted, the performance settled in at $54 \mu \mathrm{m}$ of motion for a single input wave.

The actuator performance decreased minimally but steadily over the next $8.0 \times 10^{9}$ cycles till leveling off at $48 \mu \mathrm{m}$, a common behavior in piezoceramics since the piezoelectric effect degrades with time and cycling. After $9.5 \times 10^{9}$ cycles, the behavior again became more erratic, probably due to a repeated debonding of the electrical connections. The actuator was still functional after $9.7 \times 10^{9}$ cycles when the experiment was halted - almost four orders of magnitude more than typically quoted for conventional bonded piezoceramic actuators.

Aside from the issues involving the electric leads, the actuator performed remarkable well, in addition to the observed longevity, there were no discernable cracks initiating or propagating in the actuator. The aging of the actuator was also acceptable, an $11 \%$ reduction in performance, with no ill effects seen that could be attributed to the novel DEPP grading technique. These initial results prompted further fatigue characterization studies, but bode well for the potential of FGP actuators in high-cycle applications that are currently outside the capabilities of conventional piezoceramic actuation.

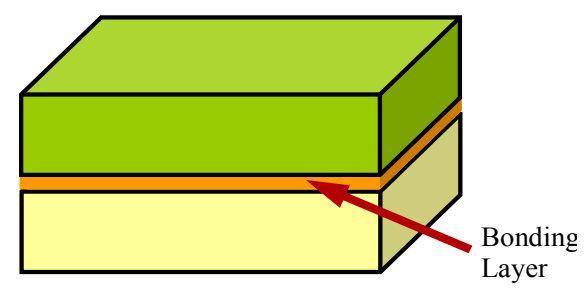

a) Discretely layered actuator

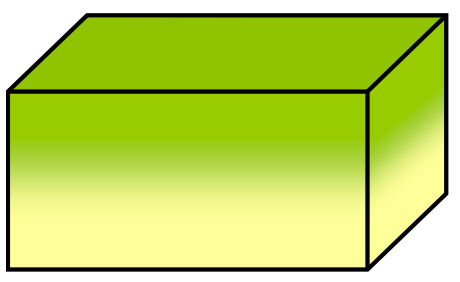

b) Continuously graded actuator $100 \mathrm{vol} \%$ PZT $80 / 20 \mathrm{vol} \% \mathrm{PZT} / \mathrm{BT}$

\section{Figure 10. Resonant Reliability} Test Specimen Construction.

\section{B. Resonance Testing}

To better gauge the performance of DEPP FGP actuators against conventionally constructed actuators, the second round of reliability tests included both FGP and layered test specimens (Fig. 10). This test was conducted at the actuators' resonant frequency, a more demanding actuation condition that could possibly be called for in commercial applications.

\section{Prototypes}

Each actuator was fabricated using the DEPP gradient constituent materials and the Micro-Fabrication by Co-eXtrusion (MFCX) process. ${ }^{29}$ The basic procedure for MFCX of FGP consists of five steps (Fig. 11): media preparation, feedrod formation, extrusion, burnout and sintering, and post processing. The FGP test specimens were

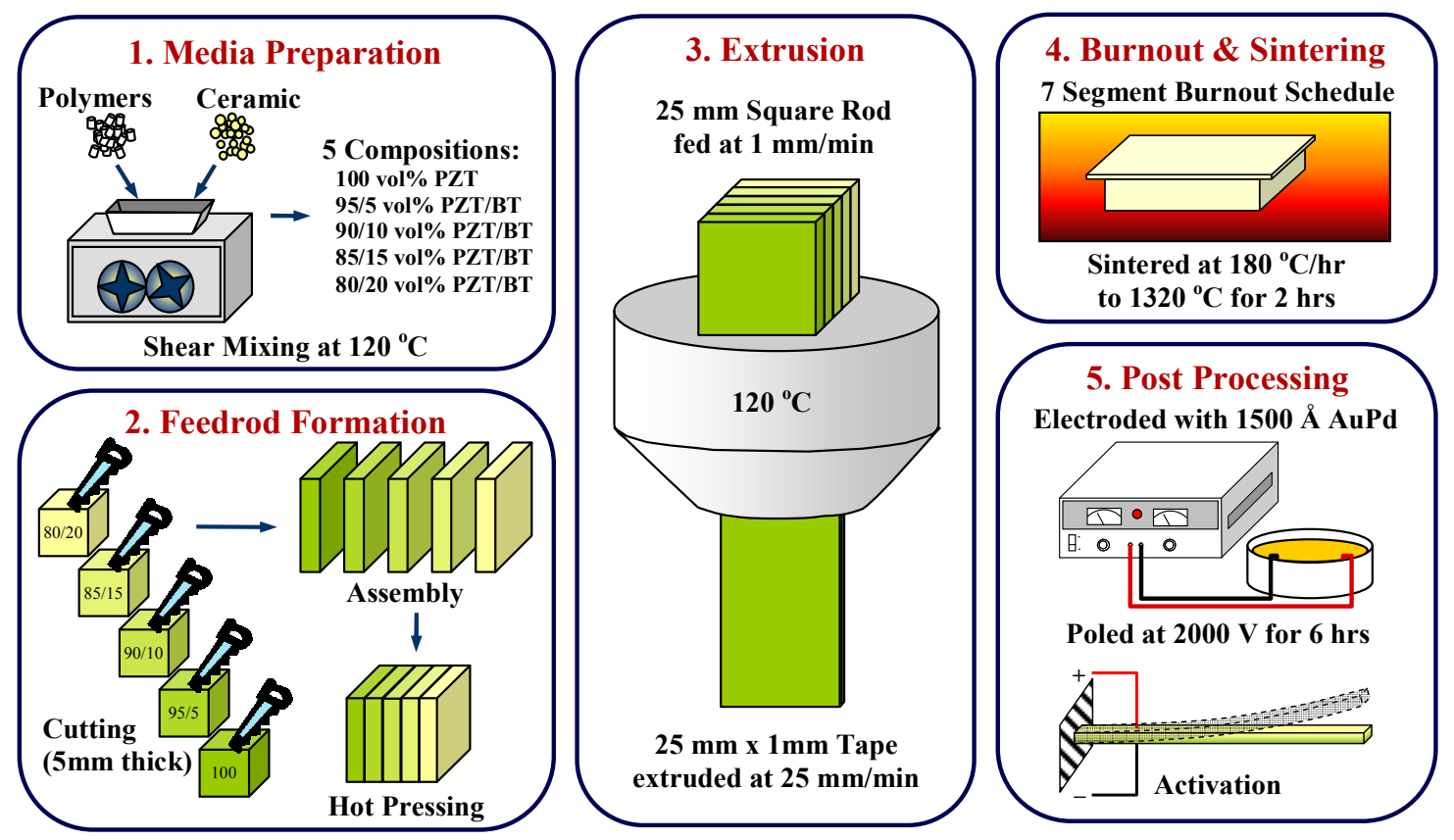

Figure 11. Overview of the MFCX Process for DEPP FGP. 
also created using the two optimal material compositions: pure PZT and 80/20 vol\% PZT/BT. Each powder was mixed with ethylene ethyle acrylate (EEA), isobutyl methacrylate (B67), polyethylene glycol, stearic acid, and heavy mineral oil in a $120{ }^{\circ} \mathrm{C}$ C.W. Brabender Instruments, Inc. PL2100 shear mixer forming a thermoplastic extrusion media. The extrusion media was assembled into an oversized version of the desired structure by forming individual feedrods of each composition, compacting the media in a $25 \mathrm{~mm}$ square die at $120^{\circ} \mathrm{C}$ under $770 \mathrm{~kg}$ load in a Bradford University Research Ltd. Extruder. The FGP feedrod was constructed by taking a $12.5 \mathrm{~mm}$ thick slice (axially) of each material, and hot pressing them into the final layered feedrod. During extrusion, this is reduced in the layered dimension from $25 \mathrm{~mm}$ square to a $1 \mathrm{~mm} \times 25 \mathrm{~mm}$ tape through a lubricated

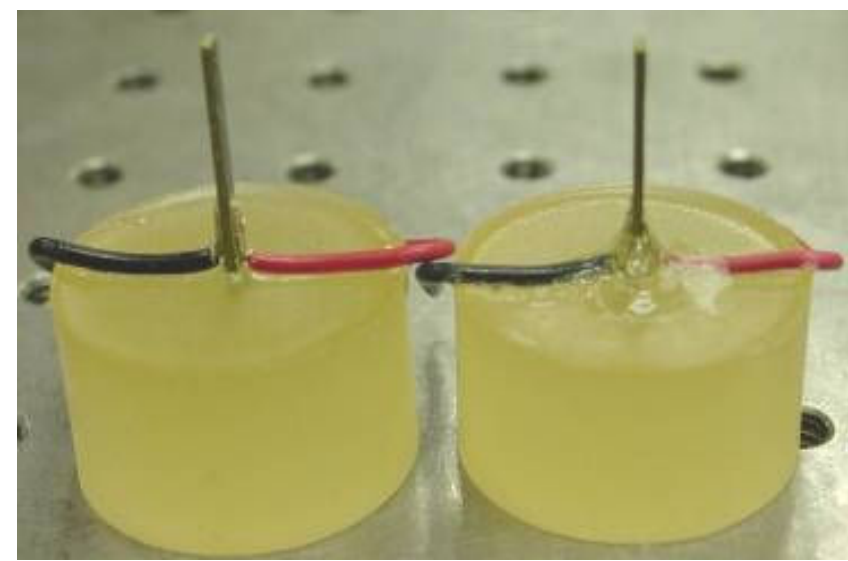

Figure 12. Resonant Reliability Test Specimens: Left - Layered $(l=20.9 \mathrm{~mm}, w=2.78 \mathrm{~mm}, t=0.78 \mathrm{~mm})$, Right - FGP $(l=20.2 \mathrm{~mm}, w=2.77 \mathrm{~mm}, t=0.81 \mathrm{~mm})$. $120{ }^{\circ} \mathrm{C}$ steel nozzle. A single extrusion run produces a large quantity of FGP tape that can be stored or reheated and cut into beam or patch shapes. The polymers in the green FGP were removed during a burnout process in a Thermolyne F48015 bench-top muffle furnace. The samples were sintered in a Micropyretics Heaters International (MHI) HIPAN-Series high temperature furnace heated at $180^{\circ} \mathrm{C} / \mathrm{hr}$ and allowed to soak for two hours at $1320^{\circ} \mathrm{C}$. The ceramic specimens were electroded on two opposing sides with $1500 \AA$ of AuPd using a Technics Hummer 6 sputtering system. The specimens were poled at room temperature in a peanut oil bath with $2000 \mathrm{~V}$ supplied by an APM 2000M Kepco DC power supply.

The FGP actuators were created by co-extruding the two materials into a $1 \mathrm{~mm}$ thick tape with equal size layers $(0.5 \mathrm{~mm})$ of each composition. The conventionally constructed two layer actuators were fabricated from individual extruded tapes of each composition. The $1 \mathrm{~mm}$ thick tapes were sintered and surface ground to a thickness equal to half that of the FGP test specimen. These layers were bonded to one another using Insulcast 501 epoxy to complete the unimorph. All actuators were sputter coated with a AuPd electrode and poled with $2000 \mathrm{~V}$. The final actuators (Fig. 12) were grouped in matched pairs with similar beam dimensions (Layered: $\quad l=20.9 \mathrm{~mm}$, $w=2.78 \mathrm{~mm}, t=0.78 \mathrm{~mm}$, FGP: $l=20.2 \mathrm{~mm}, \quad w=2.77 \mathrm{~mm}$, $t=0.81 \mathrm{~mm}) \quad$ to allow for
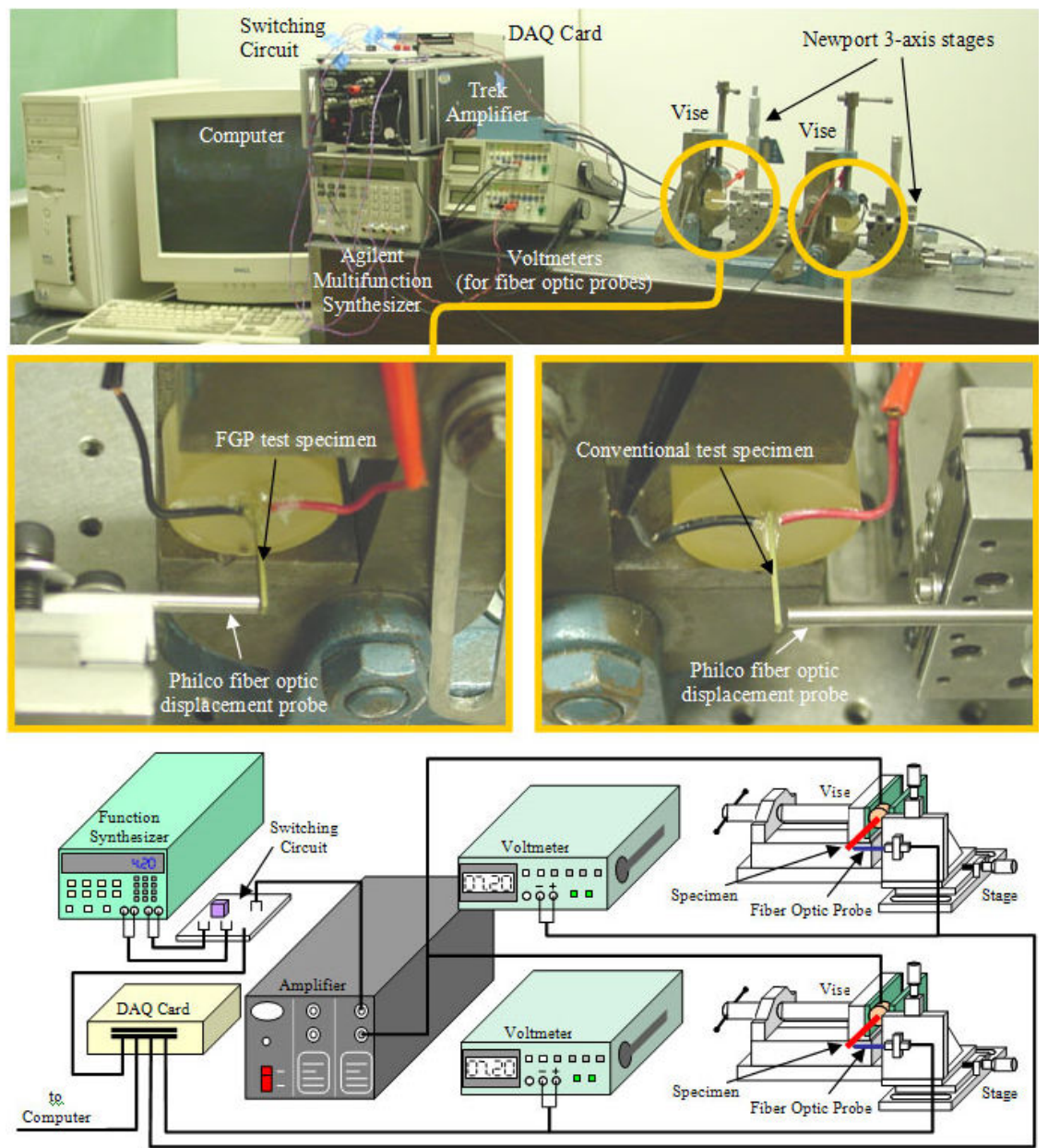

\section{Figure 13. Test Set-Up for Head-to-Head Resonant Fatigue} Characterization. 
simultaneous reliability testing at their mutual resonant frequency.

\section{Experimental Procedure}

The resonant frequency of the pair of actuators was determined by a frequency sweep using and HP 35670A digital signal analyzer and a Philtec fiber optic probe. The specimens were cycled by subjecting them to a $300 \mathrm{Vpp}$ sinusoid supplied by a dual channel Agilent 8904A multifunction synthesizer and Trek 50/750 amplifier (Fig. 13). One channel supplied a sign wave at the test frequency $(1.4 \mathrm{kHz}$ for this case) while the other generated a $1 \mathrm{~Hz}$ sine wave. Since the test frequencies exceed the bandwidth of the fiber optic probes and data acquisition devices, another LabView program was written that used a relay circuit to switch between the two driving waves so that the specimens would experience 1 million cycles at the test frequency and then be switched to the $1 \mathrm{~Hz}$ frequency where the program would measure the specimens' displacements through the fiber optic probes and record the peak measurements to a data file before switching back to the resonant frequency for additional cycling. The tests continued until over $10^{10}$ activation cycles were achieved. 3. Results

The results of the resonant fatigue test (Fig. 14) show each actuator's performance throughout the duration of the long-term experiment. Neither actuator experienced catastrophic failure over 10,809 million cycles of actuation. On the surface, these results bode well for both the conventionally constructed and graded actuators - each lasting longer than the typical $10^{6}$ cycles, but closer investigation of the trends seen in their performance tells a slightly different story.

The longer conventionally bonded actuator did initially out perform the shorter FGP specimen (approximately $14.7 \mu \mathrm{m}$ compared to $12.8 \mu \mathrm{m}$ ), but even with these advantages the difference was only $13 \%$. The results of the FGP performance tradeoff study show that this disparity could have been over $30 \%$, but was mitigated by losses within the bonding layers. It should be noted that since the resonance test actuators are significantly shorter than the below resonance actuators, their deflection ranges are much smaller. Because of this, the noise in the test data is much more prevalent than in the below resonance test, but still within acceptable levels for analysis.

The most telling results from these experiments can be seen in the chronic degradation of the conventionally constructed actuator's performance. The conventional actuator's displacement never stabilized, even after an initial 9\% drop in deflection that could be attributed to aging. Over the course of the experiment the layered specimen's deflection capabilities further diminished by $48 \%$ to $6.32 \mu \mathrm{m}$. In contrast, after aging of the piezoelectric the DEPP FGP leveled off at a consistent $9.7 \mu \mathrm{m}$, dropping by only $5 \%$ during the following $1.0 \times 10^{11}$ cycles. The bonded actuator experienced some delamination which slowly spread along the material interface as testing progressed. This scenario explains the continual drop in deflection; as more and more of the actuator became delaminated, less of the piezoelectric strain in the material was converted into out-of-plane deflection. While the DEPP FGP was fabricated with the same material and processes, it is monolithic and thus cannot delaminate, eliminating one of the primary failure mechanisms from the actuator. These experiments indicate that the DEPP FGP process improves the reliability of piezoceramic actuators by as much as four orders of magnitude.

\section{Conclusion}

This paper presents an initial study of the reliability and the associated amplification cost with DEPP FGP actuators. Numeric models for step graded actuators and analytic models for continuously graded actuators were developed to investigate the reduction in stress and displacement inherent to graded fabrication, giving insight into the potential performance/reliability tradeoffs. Simulations indicate a substantial reduction in stress $(86.2 \%)$ relative to a minimal loss of displacement capabilities $(32.5 \%)$. To further validate the premise that lifetime is increased by 
DEPP FGP fabrication, two types of fatigue experiments were conducted by cyclic activation of test specimen below resonance and at resonance. Below resonance testing subjected a bimodally graded DEPP FGP fabricated using traditional powder pressing methods to a $900 \mathrm{Vpp}$ sine wave at $500 \mathrm{~Hz}$. Prototypes demonstrated lifetimes of over $9.7 \times 10^{9}$ cycles - a four order of magnitude improvement over typically quoted conventional actuator lifetimes. Tests at resonance were conducted using a pair of geometrically similar co-extruded specimens tuned to exhibit a resonant frequency of $1.4 \mathrm{kHz}: 1$ ) a monolithic and bimodal DEPP FGP, and 2) a conventional bender created by bonding together the same two gradient materials. The specimens were cycled at resonance by a $300 \mathrm{Vpp}$ sine wave, with deflection measurements taken at $1 \mathrm{~Hz}$ every million cycles. After an initial break-in period of excitation, the performance of the DEPP FGP specimen stabilized exhibiting only a 5\% drop in performance for the duration of the test (over $1.0 \times 10^{11}$ cycles). On the other hand, the conventional bonded actuator continuously degraded, with its deflection capabilities dropping by an additional $48 \%$ as testing progressed. While the population size for these fatigue experiments was very small, these preliminary experiments indicate DEPP FGP possess great promise in overcoming the reliability limitations that have historically plagued conventional piezoceramic actuators and hindered their acceptance in commercial applications.

\section{Acknowledgments}

This research was conducted through the support of the National Science Foundation (NSF) under grant number CMS-0201031.

\section{References}

${ }^{1}$ Steel, M.R., Harrison, F., and Harper, P.G., "Piezoelectric bimorph: An experimental and theoretical study of its quasi-static response", Journal of Physics D: Applied Physics, Vol. 11, No. 6, 1978, pp. 979-989.

${ }^{2}$ Crawley E.F. and DeLuis, "Use of piezoelectric actuators as elements of intelligent structures", AIAA Journal, Vol. 25, 1987, pp. 1373-1385.

${ }^{3}$ Larson, P.H. and Vinson, J.R., "On the analysis of adaptive shell structures employing piezoelectric materials", Proceedings of the American Society of Composites $8^{\text {th }}$ technical conference, ASC, Dayton, OH, 1993, pp. 141-150.

${ }^{4}$ Paine, J.S.N., and Chaudhry, Z., "The impact of amplification on efficiency and energy density of induced strain actuators", Proceedings of ASME Aerospace Division, Vol. AD-52, ASME, New York, NY, 1996, pp. 511-516.

${ }^{5}$ Uchino, K., "Recent topics in ceramic actuators - to improve reliability and durability", IEEE $7^{\text {th }}$ International Symposium on Applications of Ferroelectrics, IEEE, New York, NY, 1990, pp.153-158.

${ }^{6}$ Uchino, K., "Reliability of ceramic actuators", IEEE International Symposium on Applications of Ferroelectrics, Vol. 2 , New York, NY, 1996, pp. 736-766.

${ }^{7}$ Badre-Alam, A., Ghandi, F., and Wang, K.W., "An improved constrained layer damping treatment for high damping low interlaminar stresses”, Proceedings of SPIE Smart Structures and Materials 2000: Damping and Isolation, Vol. 3989, SPIE, Bellingham, WA, 2000, pp. 2-13.

${ }^{8}$ Badre-Alam, A., Wang, K.W., and Ghandi, F., "An analysis of interlaminar stresses in active constrained layer damping treatments", Journal of Sound and Vibration, Vol. 269, 2004, pp. 965-990.

${ }^{9}$ Kim, H.S., Chattopadhyay, A., and Ghoshal, A., "Characterization of delamination effect on composite laminates using a new generalized layerwise approach", Computers and Structures, Vol. 81, 2003, pp. 1555-1566.

${ }^{10}$ Ghoshal, A., Kim, H.S., Chattopadhyay, A., and Prosser, W.H., "Effect of delamination on transient history of smart composite plates", Finite Elements in Analysis and Design, Vol. 41, 2005, pp. 850-874.

${ }^{11}$ Robbins, D.H. and Reddy, J.N., "Analysis of piezoelectrically actuated beams using a layer-wise displacement theory", Computers and Structures, Vol. 41, No. 2, 1991, pp. 265-279.

${ }^{12}$ Seemann, W., Straub, A., Chang, F.-K., Wolf, K., Hagedorn, P., "Bonding stresses between piezoelectric actuators and elastic beams“, Proceedings of SPIE Smart Structures and Materials 1997, Vol. 3041, SPIE, Bellingham, WA, 1997, pp. 665675.

${ }^{13}$ Mitrovic, M., Carman, G.P., and Straub, F.K., "Durability properties of piezoelectric stack actuators under combined electro-mechanical loading", Proceedings of SPIE Smart Structures and Materials 2000: Active Materials: Behavior and Mechanics, Vol. 3992, SPIE, Bellingham, WA, 2000, pp. 217-232.

${ }^{14} \mathrm{Pao}, \mathrm{Y} . \mathrm{-H}$. and Eisele, E., "Interfacial shear and peeling stresses in multilayered thin stacks subjected to uniform thermal loading", Proceedings of the ASME Winter Annual Meeting, Vol. 90-WA/EEP-34, ASME, New York, NY, 1990,pp. 1-11.

${ }^{15}$ Lee, U., Lesieutre, G.A., and Feng, L., "Safety design concerns of the piezoelectric layer applications for vibration and noise controls", Proceedings of AIAA/ASME/ASCE/HAS/ASC Structures, Structural Dynamics, and Materials ConferenceCollection of Technical Papers, Vol.1314, AIAA, New York, NY, 1997, pp.1643-1653.

${ }^{16}$ Dogan, A., Yoshikawa, S., anf Dosch, J., "Reliability studies on the bonding materials for ceramic-metal composite actuators", IEEE Journal, Vol. 3355, 1996, pp. 747-750.

${ }^{17}$ Lebedev, M. and Akedo, J., "Thick PZT film/stainless steel actuator fabricated by aerosol deposition method: fatigue property", Proceedings of SPIE Nano- and Microtechnology: Materials, Processing, Packaning, and Systems, Vol. 4936, SPIE, Bellingham, WA, 2002, pp. 345-348. 
${ }^{18}$ Wu, C.C.M., Kahn, M., and Moy, W., "Piezoelectric ceramics with functional gradients: A new application in material design", Journal of the American Ceramics Society, Vol. 79, No. 3, 1996, pp. 809-812.

${ }^{19}$ Takagi, K., Li, J.F., Yokoyama, S., Watanabe, R., Almajid, A., Taya, M., "Design and fabrication of functionally graded PZT/Pt piezoelectric bimorph actuator", Scinece and Technology of Advanced Ceramics, Vol. 3, No. 2, 2002, pp. $217-224$.

${ }^{20}$ Takagi, K., Li., J.F., Yokoyama, S., and Watanabe, R., "Fabrication and evaluation of PZT/Pt piezoelectric composites and functionally graded actuators", Journal of the European Ceraics Society, Vol. 23, 2003, pp. 1577-1583.

${ }^{21}$ Li, X., Vartuli, J.S., Milius, D.L., Aksay, I.A., Shih, W.Y., and Shih, W.H., "Electromechanical properties of a ceramic d31-gradinent flextensional actuator", Journal of the American Ceramics Society, Vol. 84, No. 5, 2001, pp.996-1003.

${ }^{22}$ Li, J.F., Takagi, K., Ono, M., Pan, W., and Watanabe, R., "Fabrication and evaluation of porous ceramics and porositygraded piezoelectric actuators", Journal of the American Ceramics Society, Vol. 86, No. 7, 2003, pp. 1094-1098.

${ }^{23}$ Zhu, X., Zhu, J., Zhou, S., Li, Q., and Liu, Z., "Microstructures of the monomorph piezoelectric ceramic actuators with functional gradients", Sensors and Actuators A: Physical, Vol. 74, 1999, pp. 198-202.

${ }^{24}$ Zhu, X., Zhu, J., Zhou, S., Li, Q., Liu, Z., Ming, N., and Meng, Z., "EPMA and TEM investigations on the interdiffusion layer of the PNN/PZT functionally gradient piezoelectric ceramics", Journal of Materials Science, Vol. 35, 2000, pp. $1031-1036$.

${ }^{25} \mathrm{Xu}$, J., Zhu, X., and Meng, Z., "Effect of the interdiffusion reaction on the compatibility in PZT/PNN functionally gradient piezoelectric materials", IEEE Transactions on Components and Packaging Technology, Vol. 22, No. 1, 1999, pp. 11-16.

${ }^{26} \mathrm{Zhu}, \mathrm{X}$., Wang, Q., and Meng, Z., "A functionally gradient piezoelectric actuator prepared by powder metallurgical process in PNN-PZ-PT system”, Journal of Materials Science Letters, Vol. 14, 1995, pp. 516-518.

${ }^{27} \mathrm{Zhu}, \mathrm{X}$. and Meng, Z., "Operational principal, fabrication and displacement characteristics of a functionally gradient piezoelectric ceramic actuator", Sensors and Actuators A: Physical, Vol. 48, 1995, pp. 169-176.

${ }^{28}$ Alexander, P.W., and Brei, D., "The design tradeoffs of linear functionally graded piezoceramic actuators", Proceedings of 2003 ASME International Mechanical Engineering Congress, Vol. 68, ASME, New York, NY, 2003, pp. 171-180.

${ }^{29}$ Alexander, P.W., Brei, D., and Halloran, J.W., "The Fabrication and Material Characterization of PZT Based Functionally Graded Piezoceramics", Proceedings of 2005 SPIE Smart Structures and Materials Conference,

${ }^{30}$ Alexander, P.W., Brei, D., and Halloran, J.W., "The Force-Deflection Behavior of Functionally Graded Piezoceramic Actuators", Proceedings of 2005 AIAA/ASME/AHS Adaptive Structures Conference, SPIE, Bellingham, WA, 2005.

${ }^{31}$ Alexander, P.W., "Dual Electro/Piezo Property Functionally Graded Piezoceramics", Ph.D. Dissertation, Department of Mechanical Engineering, University of Michigan, Ann Arbor, MI, 2006. 